\title{
Noopsicossomática em Pessoas Vivendo com HIV/AIDS: Evidências de um Modelo Explicativo
}

\author{
Alisson de Meneses Pontes \\ Faculdades Integradas de Patos \\ Patos, PB, Brasil \\ Thiago Antonio Avellar de Aquino \\ Valdiney Veloso Gouveia \\ Patrícia Nunes da Fonsêca \\ Berta Lúcia Pinheiro Kluppel \\ Universidade Federal da Paraíba \\ João Pessoa, PB, Brasil
}

\begin{abstract}
RESUMO
O objetivo deste estudo foi testar um modelo teórico proposto por Viktor Frankl, que pode explicar a dinâmica da noopsicossomática em pessoas com HIV/AIDS. Especificamente, buscou-se entender como a dimensão noológica (representada pela atitude religiosa e a realização de sentido) influencia a dimensão psíquica (afetos positivos e a percepção do passado) e, consequentemente, repercute na dimensão somática (números de células CD4+/CD8+). Participaram 115 pacientes soropositivos para o HIV/AIDS, com idade média de 39 anos (61,4\% do sexo masculino). Os resultados apontaram para a adequação deste modelo, com os índices de ajuste aceitáveis. Conclui-se que foram reunidas evidências acerca do modelo teórico da noopsicossomática em pacientes com HIV/AIDS, corroborando a relevância da dimensão noológica no processo de adoecimento e saúde.
\end{abstract}

Palavras-chave: HIV/AIDS; Espiritualidade; Afetos; Sentido da vida; Noopsicossomática.

\section{ABSTRACT}

\section{Noopsychosomatics in People Living with HIV/AIDS: Evidence of an Explanatory Model}

This study aimed to test a theoretical model proposed by Viktor Frankl, which can explain the dynamics of noopsychosomatics in people with HIV/AIDS. Specifically, we aimed to understand how the noological dimension (represented by religious attitude and sense of accomplishment) influences the mental dimension (positive affect and perception of the past), and thus affects the somatic dimension (numbers of CD4+/CD8+ cells). Participants were 115 seropositive patients for HIV/AIDS, with a mean age of 39 years $(61.4 \%$ male). Results indicate the appropriateness of this model, with acceptable fit indexes. In conclusion empirical evidence was joined about the theoretical model of noopsychosomatics in patients with HIV/AIDS, corroborating the relevance of the noological dimension in the health and disease process.

Keywords: HIV/AIDS; Spirituality; Affections; Meaning of life; Noopsychosomatics.

\section{RESUMEN}

\section{Noopsicosomática en Personas que Viven con VIH/SIDA: Evidencia de un Modelo Explicativo}

El objetivo de este estudio fue testar un modelo teórico propuesto por Viktor Frankl, que puede explicar la dinámica de noopsicosomática en personas con VIH/Sida. Específicamente, hemos tratado de entender cómo la dimensión noológica (representado por la actitud religiosa y el logro de sentido) influye en la dimensión psíquica (afecto positivo y la percepción del pasado) y, en consecuencia, afecta a la dimensión somática (número de células CD4+/CD8+). Participaron de la investigación 115 pacientes seropositivos para el VIH/sida, con una edad promedia de 39 años (61,4\% eran del sexo masculino). Los resultados apuntan la adecuación de este modelo con los índices de ajuste aceptables. Se concluyó que existen evidencias sobre el modelo teórico de noopsicosomática en pacientes con VIH/ sida, corroborando la relevancia de la dimensión noológica en el proceso de salud y enfermedad.

Palabras clave: VIH/Sida; Espiritualidad; Afectos; Sentido de la vida; Noopsicosomática. 


\section{INTRODUÇÃO}

A Síndrome da Imunodeficiência Adquirida (Acquired Imunodeficiency Syndrome/AIDS) é uma doença causada pelo Vírus da Imunodeficiência Humana (HIV), que destrói mecanismos de defesa importantes do corpo humano, provocando uma redução da resposta imune, ocasionando o aparecimento de várias doenças oportunistas (Veronesi \& Focaccia, 2010). Os principais alvos do HIV são os linfócitos T auxiliares $\mathrm{CD}^{+}$, que têm a função de memorizar e reconhecer os antígenos, bem como acionar os linfócitos T citotóxicos $\mathrm{CD}^{+}$, responsáveis por destruir os micro-organismos estranhos que invadem o corpo humano ou as células já infectadas. Após a infecção pelo HIV ocorre uma modificação na proporção dos linfócitos $\mathrm{T} \mathrm{CD} 4^{+} / \mathrm{CD}^{+}$, que em uma pessoa saudável é de duas células $\mathrm{CD} 4^{+}$ para uma $\mathrm{CD}^{+}(2 / 1)$, podendo chegar à total inversão (Balestieri, 2005).

Atualmente a AIDS é considerada uma pandemia, acometendo milhões de pessoas em todo o mundo. Estimava-se que até o final de 2011 existiriam 34 milhões de pessoas infectadas pelo HIV, prevendo neste mesmo ano a ocorrência de 2,5 milhões de novas infecções (Unaids, 2012). Nesta direção preocupante, de acordo com o Ministério da Saúde, no período de 1980 a 2012 o Brasil notificou 656.701 casos de HIV/ AIDS (Brasil, 2012), resultando em milhares de mortes. Entretanto, com o surgimento da Terapia Antirretroviral, o HIV/AIDS deixou de ser considerado uma "morte predeterminada", passando a ser classificado como uma doença crônica, o que melhorou consideravelmente a expectativa e a qualidade de vida de seus portadores (Alencar et al., 2008). Neste contexto, teve lugar o interesse por diversas variáveis de natureza social e psicológica desta patologia, visando conhecer o que poderia proporcionar maior bem-estar e qualidade de vida destas pessoas, cabendo destaque para os afetos, a religiosidade/espiritualidade e o sentido da vida.

\section{OS AFETOS NO CONTEXTO DO HIV/AIDS}

Percebe-se que a ação do HIV em seu portador vai além do comprometimento do sistema imunológico. Ademais dos sintomas físicos, os fatores psicossociais estão diretamente ligados a esta infecção, como o preconceito, o isolamento social, o medo, a culpa, a raiva e os anseios, entendendo-se que diversas destas alterações são advindas de seu diagnóstico (Marques, 2003). Ademais, segundo Melo et al. (2010), a depressão pode ser considerada o transtorno mental mais prevalente nesta população. Amâncio et al. (2010) concebem que fatores externos avaliados pelo organismo como estressores podem influenciar a predisposição para o desenvolvimento de doenças, bem como causar alterações nas funções de células imunológicas, como a diminuição na produção de citocinas e falha na regulação da resposta imune frente a diferentes patógenos, ou mesmo a morte de linfócitos responsáveis pela defesa do organismo.

Considerando o previamente exposto, concebe-se que a interação entre fatores emocionais e a função imunológica goza atualmente de evidências, como pode ser observada em áreas como a psiconeuroimunologia (PNI) e o bem-estar subjetivo (BES). A propósito, encontram-se estudos que apontam para a relação entre o estado afetivo e a saúde no que tange a eficiência imunológica (Oyanadel \& Buela-Casal, 2011; Silva Filho et al., 2012). Nesta mesma direção, Lukas (1990) afirma que um estado afetivo deteriorado enfraquece o estado imunológico do ser humano, enquanto Woods et al. (1999) ressaltam que a presença de humor deprimido, situações de perda ou depressão clínica têm sido associados com índices de supressão do sistema imunológico, como observado nos dados do Multicenter Los Angeles AIDS Cohort Study, indicando que o afeto deprimido crônico prevê declínio na contagem de $\mathrm{CD}^{+}$em homens $\mathrm{HIV}^{+}$.

Por fim, de forma análoga ao anteriormente comentado, Achterberg (1996) aponta para o elo potencial entre processos mentais positivos e a ativação do sistema imunológico. Substâncias químicas alucinógenas, como opiáceos, endorfinas e encefalinas, podem, além de produzir euforia e reduzir a dor, intensificar a resposta imune. Portanto, concebe-se que o funcionamento do sistema imune depende também do estado afetivo, que é um componente importante na avaliação da saúde geral do indivíduo (Silva Filho et al., 2012).

\section{A RELIGIOSIDADE/ESPIRITUALIDADE NO CONTEXTO DO HIV/AIDS}

A religiosidade e a espiritualidade são temas que, mesmo apresentando definições distintas, podem estar interligados, não apenas no contexto da religião, mas também quando relacionados à saúde. Koenig et al. (2001) compreendem religiosidade como o nível de envolvimento religioso e o reflexo desse envolvimento na vida da pessoa, o quanto isso influencia seu cotidiano, seus hábitos e sua relação com o mundo. Já espiritualidade pode ser definida como uma busca pessoal pela compreensão das questões últimas acerca da vida, do seu significado e da relação com o sagrado e o transcendente, produzindo autêntica experiência interior. 
Diversos autores apontam para a relação entre o estado afetivo e a religiosidade/espiritualidade em virtude de seus benefícios para a saúde mental e física (Cotton et al., 2006; Koenig et al., 2001; Panzini \& Bandeira, 2007). No entanto, Saldanha (2003) adverte que há aspectos negativos relacionados à influência da religiosidade/espiritualidade para saúde das pessoas. Lotufo Neto (2003) aponta alguns dos principais fatores acerca do caráter prejudicial da religiosidade, como a promoção de níveis patológicos da culpa, diminuição da autoestima, estabelecimento de uma base para reprimir a raiva, intensifica a ansiedade e o medo por meio de crenças punitivas, inibe a expressão de sensações sexuais e abre o caminho para o desajuste sexual, e encoraja a visão de que o mundo é dividido entre "santos" e "pecadores". Apesar do anteriormente comentado, Moreira-Almeida, Lotufo Neto e Koenig (2006) descreveram que de 850 pesquisas que abordaram esta relação, a maioria endossou a associação do envolvimento religioso com maiores níveis de satisfação com a vida, felicidade, afeto positivo, moral mais elevada, senso de propósito e significado da vida, bem-estar, otimismo e esperança; contrariamente, registraram-se menores índices de depressão, ansiedade e abuso de substâncias. Neste sentido, justifica-se abordar o papel da religiosidade/espiritualidade para a saúde de pessoas que vivem com HIV/AIDS.

A temática da religiosidade/espiritualidade e saúde tem ocupado lugar de destaque no cenário mundial nos últimos anos, considerando questões como adesão a tratamentos médicos e psicológicos (Ferreira et al., 2012), enfrentamento de doenças (Panzini \& Bandeira, 2005), sentido da vida (Aquino et al., 2009), suporte social (Seidl et al., 2005), melhor saúde física e mental (Koenig et al., 2001; Moreira-Almeida et al., 2006) e menor probabilidade de desenvolver comportamentos de risco, como abuso de drogas/álcool, delinquência ou crime (Moreira-Almeida et al., 2006; Panzini \& Bandeira, 2007), e também na diminuição de sintomas depressivos em pessoas que padecem do HIV/AIDS (Koenig et al., 2001). Neste sentido, Terrin (1998) atribui à religião uma força terapêutica, infundindo no homem confiança, atitude de pensar positivamente, confiar em Deus e contemplar o mundo com otimismo. Portanto, a religião exerce influência positiva em estados de espírito, psique e humores de uma pessoa. Em vista disso, percebe-se que a religiosidade e a espiritualidade são variáveis que, mesmo apresentando definições distintas, podem estar interligadas, não apenas na perspectiva do sagrado, mas também quando relacionadas com a saúde.

De acordo com Saroglou (2003), a espiritualidade/ religiosidade pode emergir nas pessoas como uma forma de enfrentar as crises ou adversidades, a exemplo do surgimento de uma doença crônica (a AIDS é um caso típico). Desta forma, realizaram-se pesquisas avaliando a importância da espiritualidade/ religiosidade para a saúde de pessoas que vivem com HIV/AIDS. Um dos estudos, de cunho longitudinal, levado a cabo nos Estados Unidos, analisou oito medidas de espiritualidade/religiosidade (e.g., Escala de Terapia-Espiritualidade Expandida, Coping Positivo e Negativo e Índice de Religião de Duck) que foram respondidas por 450 portadores de HIV/AIDS de três cidades (Cotton et al., 2006). Os resultados demonstram que maiores níveis de espiritualidade estão relacionados com maior satisfação com a vida, indicadores favoráveis de saúde, qualidade de vida, bem-estar geral, suporte social, autoestima e otimismo, assim como menos sintomas depressivos. Deste contingente, 339 pacientes $(75 \%)$ disseram ter a fé pelo menos um pouco fortalecida, utilizando estratégias de enfrentamento religioso positivo.

Por fim, sabe-se que um dos benefícios da religiosidade/espiritualidade é encontrar sentido para a vida em contextos de saúde, o que ocorre por meio da busca pelo sagrado e transcendente (Moreira-Almeida, Lotufo Neto, \& Koenig, 2006; Saroglou, 2003). Nesta perspectiva, Aquino et al. (2009) verificaram a correlação entre a religiosidade/espiritualidade e o sentido da vida em 300 pessoas. Seus resultados indicaram que a atitude religiosa se correlacionou $(p<0,001)$ positivamente com a realização existencial $(r=0,36)$, fazendo-o negativamente com o desespero $(r=-0,26)$ e o vazio existencial $(r=-0,19)$. Desta forma, concluiu-se que a atitude religiosa é uma forma de encontrar o sentido da vida, assim como um elemento de prevenção de vazio e desespero existenciais. Deste modo, parece relevante ter em conta o sentido da vida quando considerados os pacientes com HIV/AIDS.

\section{A NOOPSICOSSOMÁTICA NO CONTEXTO DO HIV/AIDS}

Espírito Santo (2011) comenta que a religiosidade e a espiritualidade ganham importância por meio de fatores como o encontro de sentido para a vida e de razão para viver após o diagnóstico de uma doença grave, possibilitando que o indivíduo entenda, interprete e viva a doença de modo que encontre seu significado. Assim, demanda-se compreender a importância do sentido da vida para quem vive com HIV/AIDS. No caso, a busca pelo sentido da vida é uma atitude natural e uma característica distintiva do ser humano, pois somente ele é capaz de questionar a própria existência e discutir a problemática do ser (Frankl, 2012). Neste 
contexto, um dos autores principais acerca da busca de sentido foi Viktor Frankl (1905-1997); ele criou a Logoterapia, que tem como função a cura da alma por meio de método fundado no "encontro" e na conquista da liberdade. Esta, por sua vez, conduz o homem a descobrir o sentido último de cada ação e de toda sua existência (Frankl, 2009).

A Logoterapia aborda o ser humano de forma integral, considerando-o em sua totalidade somática, psíquica e noológica (espiritual). Esta última compreende todas as qualidades que diferenciam o homem dos demais animais, entendendo o espiritual não apenas como dimensão religiosa, mas valorativa, intelectual e artística, apresentando características como a livre tomada de decisões, a consciência moral e a criatividade (Lukas, 1989). Desta forma, Frankl (1992) concebe que há uma relação entre estas três dimensões na saúde, denominada de noopsicossomática. Sua configuração resulta na proposta por ele apresentada de que a situação imunológica está relacionada com a situação afetiva, a qual também está associada à dimensão noológica. Assim sendo, a orientação do homem para o sentido seria um fator não apenas de proteção à saúde, mas de sobrevivência.

Em pesquisa realizada com 46 pacientes soropositivos para o HIV, Litwinczuk e Groh (2007) avaliaram a relação entre espiritualidade, propósito da vida e bem-estar. Seus resultados demonstraram que houve uma correlação positiva entre o sentido da vida e a religiosidade $(r=0,29, p<0,05)$. Estes autores concluíram que o sentido foi um dos fatores protetores para saúde, oferecendo suporte para a teoria frankliana. De fato, esta interação da noopsicossomática pode ser realmente decisiva, como aponta Frankl (2008) ao descrever que os prisioneiros que tinham mais possibilidades de sobreviver nos campos de concentração nazistas eram aqueles que estavam norteados por um sentido pelo qual foram compelidos.

Lukas (1990) reforça a ideia de que quando não há uma plenitude de sentido, tanto a saúde psíquica quanto a física são colocadas em risco. Portanto, a perda da realização de sentido abala o psiquismo e, consequentemente, um estado afetivo deteriorado enfraquece o sistema imunológico do ser humano. Esta concepção é coerente com a fundamentação de Frankl (2012), quem propõe um modelo que considera, além do psicofísico, a dimensão noológica, que na maior parte das vezes é ignorada pelas áreas da saúde, abordando o homem em sua totalidade.

A justificativa para este modelo está evidenciada na própria sociedade contemporânea, que nas últimas décadas deixou de vivenciar valores mais intrínsecos (Kasser \& Ahuvia, 2002; Kasser \& Ryan, 1993), abandonando igualmente as tradições e fracassando na realização de sentido, o que resultou na busca do prazer como fim para a felicidade, reforçando a neurose de massa que é o vazio existencial. Consequentemente, há uma repercussão drástica para o estado emocional, que resulta em redução na capacidade de defesa do sistema imune (Lukas, 1990). Compreende-se, portanto, que a visão de Frankl (1992) sobre o homem, evidenciada na dinâmica da noopsicossomática, surge como fator relevante para a saúde. Desta forma, a presente pesquisa procurou testar empiricamente a relação entre as dimensões noológica, psíquica e somática na saúde de pessoas que vivem com HIV/AIDS.

Especificamente, o presente estudo pretendeu conhecer em que medida e direção se relacionam a atitude religiosa, a realização de sentido, os afetos e a percepção do passado, procurando elaborar e testar um modelo explicativo que considera como variável critério o nível de $\mathrm{CD}^{+} / \mathrm{CD}^{+}$destes pacientes soropositivos.

\section{MÉTODO}

\section{Participantes}

Participaram da pesquisa 115 pacientes soropositivos para o HIV/AIDS, com idades variando entre 18 a 63 anos $(m=39,92, d p=10,32)$, a maioria do sexo masculino $(61,4 \%)$, solteira $(54,8 \%)$ e empregada $(61,7 \%)$, recebendo até dois salários mínimos $(83,5 \%)$ e indicando ter uma religião definida ( $88 \%)$. Tratou-se de uma amostra de conveniência, sendo as pessoas convidadas a participar voluntariamente do estudo. Foram excluídos 17 participantes pela impossibilidade de acesso aos seus prontuários, decorrente de informações incompatíveis fornecidas pelos próprios pacientes (por exemplo, o nome e/ou o número do prontuário).

\section{Instrumentos}

Os pacientes responderam um livreto com cinco instrumentos:

1 - Questionário de Sentido de Vida (QSV). Originalmente construída em inglês por Steger et al. (2006) e adaptada ao contexto brasileiro por Aquino, Gouveia, Pontes, Pereira, \& Fernandes (no prelo). Trata-se de versão com dez itens dispostos em uma escala de 7 pontos, variando de $\mathbf{1}=$ Totalmente falso a $\mathbf{7}=$ Totalmente verdadeiro. As consistências internas na presente amostra foram: realização de sentido: $\alpha=0,73$ (4 itens, e.g., Eu compreendo o sentido da minha vida) e busca de sentido: $\alpha=0,79$ (6 itens e.g., Eu estou procurando alguma coisa que faça com que minha vida tenha sentido).

2 - Escala de Percepção Ontológica do Tempo (EPOT). Esta escala foi desenvolvida por Aquino (2009). Compõe-se de dez itens dispostos em uma 
escala de 5 pontos, variando de $\mathbf{1}$ (Discordo totalmente) a 5 (Concordo totalmente). As consistências internas de cada fator na amostra em questão foram: passado: $\alpha=0,44$ (3 itens, e.g., Ao olhar para o passado, sinto-me realizado com o que alcancei), presente: $\alpha=0,70$ (3 itens, e.g., Ao olhar para o presente, vejo sempre um motivo para estar no mundo) e futuro: $\alpha=0,72$ ( 3 itens, e.g., Ao olhar para o futuro, vejo muitas possibilidades de escolha).

3 - Escala de Afetos Positivos e Negativos (EAPN). Este instrumento foi elaborado por Diener e Emmons (1984) para avaliar a valência dos afetos, apresentando índices psicométricos satisfatórios. A versão brasileira (Chaves, 2003) está composta por dez itens dispostos em uma escala de resposta de 7 pontos, variando de $\mathbf{1}$ (Nada) a 7 (Extremamente). As consistências internas da amostra foram: afetos positivos: $\alpha=0,82$ (3 itens e.g., Feliz, Otimista) e afetos negativos: $\alpha=0,79$ (3 itens, e.g., Deprimido, Infeliz).

4 - Escala de Atitude Religiosa (EAR-20). Construída por Aquino, Gouveia, Silva, \& Aguiar (2013) é composta por 20 itens dispostos em uma escala de cinco pontos, variando de 1 (Nunca) a 5 (Sempre). As consistências internas na amostra atual são apresentadas a seguir: afetivo: $\alpha=0,60$ (3 itens: e.g., Extravaso a tristeza ou alegria através de músicas religiosas), comportamental: $\alpha=0,81$ (5 itens: e.g., $A$ religião/religiosidade influencia nas minhas decisões sobre o que eu devo fazer), cognitivo: $\alpha=0,84$ (7 itens, e.g., Leio as escrituras sagradas: biblia ou outro livro sagrado) e corporeidade: $\alpha=0,88$ (5 itens, e.g., Costumo levantar os braços em momentos de louvores).

5 - Questionário demográfico e clínico. Visou caracterizar os participantes, considerando as seguintes perguntas: sexo, idade, grau de escolaridade, renda familiar, situação conjugal, local de residência e dados clínicos (tempo de diagnóstico da infecção, tratamento medicamentoso, proporção de células $\mathrm{CD}^{+} / \mathrm{CD}^{+} \mathrm{e}$ existência de doenças oportunistas).

\section{Procedimento}

Foram contatados individualmente os pacientes, não internados, que se encontravam em consulta médica periódica no Serviço de Atendimento Especializado situado em um hospital referência no tratamento de doenças infectocontagiosas. Convidados a participar, os que concordaram tiveram que assinar termo de consentimento livre e esclarecido. $\mathrm{Na}$ ocasião, concordaram também em permitir o acesso aos seus prontuários clínicos. Em média, a duração de sua participação foi de 40 minutos. Ressalta-se que este estudo seguiu as normas que regem a pesquisa envolvendo seres humanos, como prescreve a
Resolução CNS n $466 / 12$, sendo o projeto aprovado pelo Comitê de Ética do Hospital Universitário Lauro Wanderley, da Universidade Federal da Paraíba (Protocolo de n⿳⺈ 712/10).

\section{Análise dos dados}

O SPSS (versão 18) foi empregado para realizar análises descritivas (médias, desvios padrões, frequências e porcentagens) para caracterização dos participantes, análises bivariadas [correlações de Pearson (r)] com o fim de verificar associações entre as variáveis e Anova para comparar o índice CD4+/CD8+ em razão do tempo de diagnóstico dos pacientes [grupos: "até 1 anos" $(n=23)$, "1 a 2 anos" $(n=22)$, "3 a 4 anos" $(n=12)$ e "5 ou mais anos" $(n=58)]$. Por meio do AMOS (versão 7) foi efetuada uma path analysis (análise de caminhos) para testar o modelo teórico de predição dos índices dos linfócitos representados pela razão $\mathrm{CD}^{+} / \mathrm{CD}^{+}$. A escolha desta proporção se justifica pelo fato de a contagem de células $\mathrm{T} \mathrm{CD}^{+}$não ser suficiente para efetuar o prognóstico e estabelecer o seguimento das terapias antirretrovirais (Arroz et al, 2001). Por outro lado, a proporção $\mathrm{CD} 4^{+} / \mathrm{CD} 8^{+}$se apresenta como um parâmetro laboratorial avaliativo do funcionamento do sistema imunológico, constituindo-se em um indicador de maior morbidade e mortalidade (Serrano-Vilar, et al. 2014). O ajuste do modelo foi avaliado levando em conta os indicadores recomendados pela literatura (Byrne, 2010).

\section{RESULTADOS}

Em relação à caracterização clínica, foi verificado o tempo de diagnóstico, onde $20 \%$ dos pacientes adquiriram o vírus há no máximo um ano; $19,1 \%$ entre um e dois anos; $10,4 \%$ de três a quatro anos; e $50,4 \%$ de cinco anos acima. Quanto ao uso dos antirretrovirais, 90,4\% afirmaram tomar a medicação regularmente. Ressalta-se que $50 \%$ apresentaram doenças oportunistas, sendo as mais prevalentes a tuberculose $(17,4 \%)$, a pneumonia $(9,6 \%)$ e o herpes $(9,6 \%)$. Quanto à contagem dos linfócitos $\mathrm{T}$, verificou-se que os $\mathrm{CD}^{+}$, um dos indicadores das condições do sistema imunológico, tiveram uma amplitude de $23 / \mathrm{mm}^{3}$ a $1757 / \mathrm{mm}^{3}$ (número de células por milímetro cúbico de sangue). No tocante aos linfócitos $\mathrm{T} \mathrm{CD} 8^{+}$, o mínimo foi de $279 / \mathrm{mm}^{3}$ e o máximo de $2887 / \mathrm{mm}^{3}$. Referente à proporção entre as células $\mathrm{CD}^{+} / \mathrm{CD}^{+}$, constatou-se variação de 0,03 a 2,39; apenas $11,3 \%$ dos pacientes apresentaram o índice $\mathrm{CD} 4^{+} / \mathrm{CD}^{+}$maior que um.

Inicialmente, efetuou-se uma Anova considerando como variável independente o tempo de diagnóstico dos pacientes com HIV/AIDS e variável dependente 
o índice $\mathrm{CD}^{+} / \mathrm{CD}^{+}(F=3,68, p<0,01)$. Quando a relação $\mathrm{CD} 4+/ \mathrm{CD} 8+$ foi avaliada em função do tempo de diagnóstico, os grupos de "3 a 4 anos" apresentaram valores semelhantes aos que tinham diagnóstico de "5 ou mais anos", e ambos apresentaram, dentro do grupo, os níveis mais próximos do padrão considerado saudável ( 2 células $\mathrm{CD}^{+}$para $1 \mathrm{CD}^{+}$). No caso, como não foi possível assumir homogeneidade de variâncias [Teste de Levene $=4,09, p<0,01$ ], efetuou-se o teste post hoc de Tamhame, que apontou diferença quanto à relação das células $\mathrm{CD} 4+/ \mathrm{CD} 8$ entre os pacientes que possuíam tempo de diagnóstico de "até 1 ano" $(m=0,35, d p=0,21)$ e aqueles com "5 ou mais anos" $(m=0,67, d p=0,46)$.

Posteriormente foram verificadas as correlações entre as variáveis atitude religiosa, percepção ontológica do tempo, afetos e sentido da vida, que são apresentadas na Tabela 1. Conforme se observa nesta tabela, é possível destacar as correlações positivas entre os fatores que compõem o modelo da noopsicossomática em pessoas que vivem com HIV/AIDS, a saber: os quatro componentes da atitude religiosa se associaram diretamente com o fator realização de sentido, sendo que este último o fez com os afetos positivos, que, por sua vez, se correlacionaram com a percepção positiva do passado. Portanto, estes resultados endossam o modelo proposto na presente pesquisa. Constatouse, ainda, que o índice $\mathrm{CD}^{+} / \mathrm{CD}^{+}$dos pacientes em estudo se correlacionou unicamente como o seguinte item do EPOT: "Ao olhar para o passado, sinto-me realizado com o que já alcancei” ( $r=0,22, p<0,05)$.

Por fim, levando em conta a noopsicossomática proposta pela Logoterapia e Análise Existencial (Frankl, 2012), decidiu-se testar um modelo explicativo em que a dimensão noética (atitudes religiosas e realização de sentido) influencia a dimensão psicológica (afetos positivos e percepção do passado), que, por sua vez, explica a dimensão somática (linfócitos $\mathrm{T} \mathrm{CD} 4^{+} / \mathrm{CD}^{+}$) em pessoas que vivem com HIV/AIDS. No caso, submeteu-se o modelo à modelagem de equações estruturais, sendo que os resultados, no geral, apoiaram a adequação deste modelo $\left[\chi^{2}(20)=27,40, p<0,01\right.$; $\chi^{2} / \mathrm{gl}=1,37, G F I=0,95, A G F I=0,91, C F I=0,97 \mathrm{e}$ RMSEA $=0,06(I C 90 \%=0,00-0,11)] ;$ o teste Pclose indicou que o valor do RMSEA não é estatisticamente superior ao tomado como ponto de corte, isto é, $0,05(p=0,38)$. Ressalta-se também que todas as saturações (os lambdas) foram estatisticamente diferentes de zero $(\lambda \neq 0 ; z>1,96, p<0,05)$. O modelo correspondente é apresentado na Figura 1 a seguir.

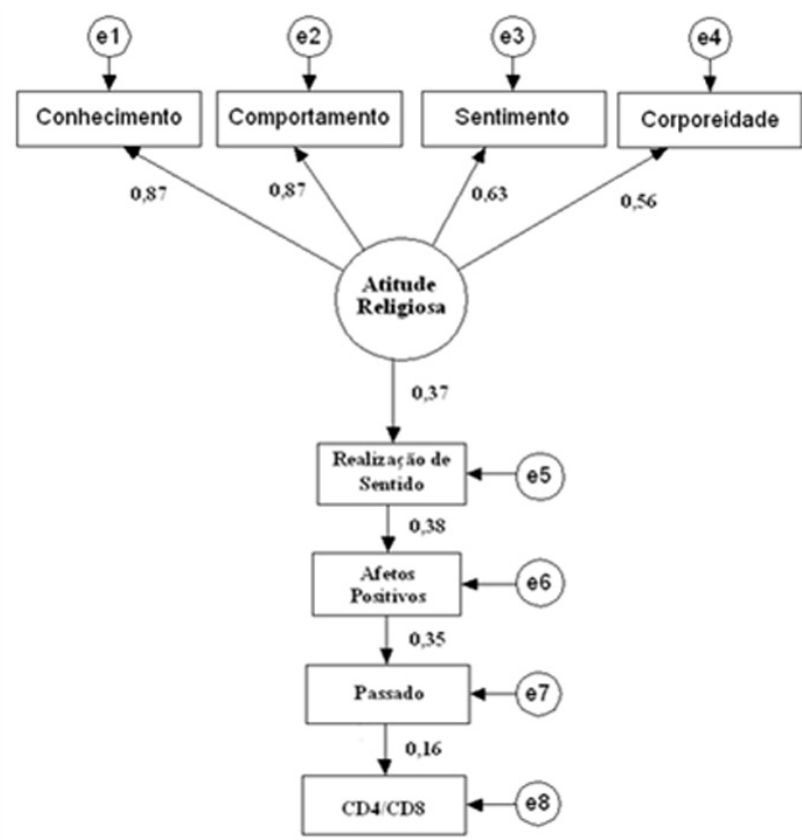

Figura 1. Modelo teórico para explicar a noopsicossomática em pacientes com HIV/AIDS

TABELA 1

Correlações entre a Atitude Religiosa, Percepção Ontológica do tempo, Afetos e Sentido da Vida

\begin{tabular}{|c|c|c|c|c|c|c|c|c|c|c|}
\hline 2 & $0,20 *$ & & & & & & & & & \\
\hline 3 & 0,07 & $0,73 * *$ & & & & & & & & \\
\hline 4 & $0,35 * *$ & $0,54 * *$ & $0,50 * *$ & & & & & & & \\
\hline 5 & 0,00 & $-0,22 *$ & $-0,24 * *$ & $-0,39 * *$ & & & & & & \\
\hline 6 & 0,03 & $0,26 * *$ & $0,27 * *$ & $0,25^{* *}$ & $-0,13$ & & & & & \\
\hline 7 & 0,14 & $0,23^{*}$ & $0,24 * *$ & $0,25^{* *}$ & 0,01 & $0,76^{* *}$ & & & & \\
\hline 8 & 0,10 & $0,24 * *$ & $0,32 * *$ & $0,32 * *$ & $-0,06$ & $0,49 * *$ & $0,45 * *$ & & & \\
\hline 9 & $-0,01$ & 0,16 & $0,22 *$ & 0,17 & $-0,03$ & $0,51 * *$ & $0,56^{* *}$ & $0,49 * *$ & & \\
\hline 10 & $0,23^{*}$ & $0,49 * *$ & $0,53 * *$ & $0,38 * *$ & $-0,31^{* *}$ & $0,36 * *$ & $0,35^{* *}$ & $0,23 *$ & $0,25 * *$ & \\
\hline 11 & 0,24 & 0,10 & 0,04 & $-0,72$ & 0,18 & $-0,22 *$ & $-0,06$ & 0,03 & $-0,10$ & $-0,18^{*}$ \\
\hline Variáveis & 1 & 2 & 3 & 4 & 5 & 6 & 7 & 8 & 9 & 10 \\
\hline
\end{tabular}

$* p<0,05 ; * * p<0,01$.

Identificação das variáveis: 1 = Passado, 2 = Presente, $3=$ Futuro, $4=$ Afetos Positivos, $5=$ Afetos Negativos, $6=$ Conhecimento Religioso, $7=$ Comportamento Religioso, 8 = Corporeidade Religiosa, 9 = Sentimento Religioso, 10 = Realização de Sentido, 11 = Busca de Sentido. 


\section{DISCUSSÃO}

No tocante a semelhança na proporção de $\mathrm{CD}^{+} /$ $\mathrm{CD}^{+}$entre os grupos que possuíam tempo de diagnóstico de "3 a 4 anos" e "5 ou mais anos", observa-se que este resultado pode ser atribuído ao maior tempo de administração da medicação adequada (Geocze et al., 2010) e maior adaptação emocional e social à doença. Já a diferença de $\mathrm{CD}^{+} / \mathrm{CD}^{+}$entre os grupos de "até 1 ano" e "5 ou mais anos", pode ser compreendida a partir dos impactos físicos [administração da medicação adequada e adaptação aos efeitos colaterais (Geocze et al., 2010) e alta viremia (Balestieri, 2005)] e emocionais [choque, medo ansiedade, frustração e culpa (Remor, 2002)], comumente vivenciadas no início do quadro HIV/AIDS. Associado a estes fatores está a tendência mundial de diagnóstico tardio nesta população, incluindo no Brasil (UNAIDS, 2012), que tende a levar os portadores do vírus a procurarem ajuda já com o comprometimento do seu sistema imunológico.

No presente estudo foi verificada uma relação direta entre os quatro fatores da Atitude Religiosa e a sensação de realização de sentido. Resultado semelhante foi encontrado por Aquino et al. (2009). Nesta direção, Fryback e Reinert (1999) relatam em seu estudo, considerando pessoas soropositivas para o HIV, que os indivíduos percebiam a espiritualidade como a ponte entre a desesperança e o sentido na vida. A espiritualidade e a religiosidade surgem como fatores importantes em pacientes que lidam com doenças infecciosas crônicas ou que estão no final da vida (Cotton et al., 2006), constituindo-se como fonte de apoio social relevante para eles e suas famílias (Silva et al., 2009), configurando-se como uma estratégia importante de enfrentamento da doença (Resende et al., 2008). Ademais, a religiosidade pode exercer efeitos psico-higiênicos na medida em que pode ajudar o ser humano a encontrar um sentido na vida (Frank1, 2009).

Observou-se que a realização de sentido se associou diretamente com os afetos positivos. Alguns autores já apontaram que o estado afetivo está associado ao bom funcionamento do sistema imunológico (Leserman, 2008; Linares et al., 2008; Silva Filho et al., 2012). Frankl (2011) afirma que o ser humano necessita de um fundamento transcendente para a felicidade, posto que carece de um motivo ou uma razão para ser feliz, tendo os afetos positivos um efeito da realização de sentido. Por outro lado, quanto mais o homem a busca como um fim em si mesmo, mais se sentirá frustrado existencialmente. Desta forma, o vazio existencial se manifesta por meio do tédio, da falta de interesse e da indiferença, o que pode ocasionar transtornos psicossociais diversos, como agressão e depressão. Nesta direção, o estudo de Steger et al. (2006) apontou uma correlação positiva entre presença de sentido e satisfação com a vida, isto é, quanto maior a presença de sentido na vida, maior sua satisfação e melhores os afetos experimentados.

Também foi possível constatar correlação direta entre os afetos positivos e a percepção do passado em portadores de HIV/AIDS. Supõe-se que os afetos condicionam a visão do passado, pois os pacientes que apresentaram maiores índices de afetos positivos relataram uma percepção positiva do passado. Frankl (2012) concebe que o passado seria a dimensão mais segura do ser humano, pois nele o homem preserva todos os valores realizados em sua existência; configura-se como o que foi realizado, estando preservado. Relacionando a perspectiva de passado com os afetos, Frankl (1992) considera que, no caso de depressão ou "baixa vital", ocorreria uma redução no estado emocional que, consequentemente, poderia causar alteração na forma como se percebe o passado. Este autor descreve que é como se o paciente compreendesse seu passado por meio de uma lupa aumentada e enviesada, distorcendo sua percepção. Knapp (2004), por sua vez, concebe que, apesar das dificuldades de memória, pacientes depressivos se lembram bem de eventos negativos.

Na direção do anteriormente comentado, Bower (1981) observou que o estado emocional está diretamente associado com a memória; assim, um estado afetivo deprimido a distorce, evocando seletivamente exemplos de fracasso no passado. Por outro lado, um estado positivo de humor se associa com uma melhor perspectiva do passado. Pergher et al. (2006) denominam esta relação de MoodCongruent Memory (MCM), que pode ser definida como a tendência de codificar ou recordar materiais que possuem valência consistente com o estado afetivo em que a pessoa se encontra. Portanto, um indivíduo que experimenta alegria codifica e/ou recorda mais facilmente e em maior número as informações que contenham afetos positivos. Por fim, percebeu-se que o sistema imunológico, aferido pela relação CD4+/CD8+, foi predito pelo fator percepção do passado. Desta forma, concebe-se que a forma como o paciente percebe seu passado pode repercutir no funcionamento de seu sistema imunológico.

Corroborando o que se comentou, Nauert (2011) destaca que a atitude das pessoas sobre eventos passados repercute em sua saúde e qualidade de vida. Deste modo, evocar lembranças do passado em um contexto positivo pode ser um fator que favorece melhor estado de saúde. De fato, em estudo desenvolvido por 
Hamilton et al. (2003), considerando pessoas com problemas cardíacos, observou-se que a percepção positiva do passado gerou condutas saudáveis e de proteção para a saúde. É possível que isso se aplique para outros problemas de saúde enfrentados pelas pessoas, como asseveram Oyanadel e Buela-Casal (2011), os quais descrevem que aquelas orientadas por uma visão negativa do passado apresentam indicadores piores em todos os domínios de saúde física e mental. Esta associação entre a visão do passado e o estado de saúde pode justificar o fato de o índice $\mathrm{CD} 4^{+} / \mathrm{CD} 8^{+}$dos pacientes em estudo ter se correlacionado unicamente com o item do EPOT, pois este enfoca precisamente o passado.

\section{CONSIDERAÇÕES FINAIS}

Estima-se que este estudo oferece evidências empíricas acerca da adequação do modelo teórico da noopsicossomática em pacientes com HIV/AIDS, cujos indicadores de ajuste foram adequados (Byrne, 2010). Neste sentido, espera-se que seus objetivos tenham sido alcançados. Considerando o exposto, sugere-se que a religiosidade/espiritualidade, o sentido da vida e os afetos são variáveis presentes no contexto da infecção pelo HIV/AIDS. Deste modo, este estudo buscou testar o modelo proposto por Frankl (2012), explicando a dinâmica da noopsicossomática em pessoas com este quadro de doença. Nesta oportunidade, há que ressaltar que não se pretendeu reduzir as dimensões supracitadas aos fatores aqui utilizados, tendo em vista que são construtos bastante amplos e complexos.

De forma semelhante, cabe assinalar que não foi o propósito deste estudo generalizar os resultados, mas testar empiricamente a adequação do modelo teórico de Frankl (2012) sobre a noopsicossomática no contexto da saúde. Reconhece-se, neste contexto, que a amostra foi relativamente reduzida e específica, sendo de conveniência. Entretanto, estas limitações não invalidam os achados, que se mostraram consistentes com o marco teórico considerado. Neste sentido, evidencia-se a contribuição da pesquisa ao mostrar a relevância de fatores como religiosidade/espiritualidade e sentido da vida no contexto do HIV/AIDS, quiçá extensiva para a saúde como um todo. Esta percepção confronta, sobretudo, a atual abordagem prestada por alguns profissionais de saúde, que negligenciam tais fatores.

Por fim, considera-se importante replicar este estudo, por exemplo, ampliando a amostra ou considerando outro contexto da saúde, o que permitirá avaliar o escopo do modelo testado. Também seria possível pensar em portadores de HIV em diferentes estágios, incluindo os internados, de modo a avaliar a consistência dos achados previamente descritos. Diante dos achados, ressalta-se a necessidade de políticas públicas que englobem não apenas os determinantes psicossociais e clínicos relacionados ao contexto do HIV/AIDS, mas também aqueles que tratam o paciente de forma integral, incluindo a dimensão genuinamente humana, isto é, a noética. Apesar de perdas advindas de uma doença crônica, o ser humano possui ainda a capacidade de transcender sua própria condição somática por meio de um encontro de sentido no próprio sofrimento, muitas vezes oferecida por um sistema religioso. Portanto, concebe-se que o sentido se constitui como uma condição para a saúde em pessoas com HIV/AIDS.

\section{REFERÊNCIAS}

Achterberg, J. (1996). A imaginação na cura: xamanismo e medicina moderna (Trad. Carlos Eugênio Marconsde de Moura). São Paulo: Summus.

Alencar, T. M. D., Nemes, M. I. B., \& Velloso, M. A. (2008). Transformações da "aids aguda" para a "aids crônica": percepção corporal e intervenções cirúrgicas entre pessoas vivendo com HIV e AIDS. Ciência \& Saúde Coletiva, 13, 1841-1849. http://dx.doi.org/10.1590/S1413-81232008000600019

Aquino, T. A. A. (2009). Atitudes e intenções de cometer suicídio: Seus correlatos existenciais e normativos. Tese de Doutorado. Departamento de Psicologia, Universidade Federal da Paraíba, João Pessoa, PB.

Aquino, T. A. A. (2013). Logoterapia e análise existencial: Uma introdução ao pensamento de Viktor Frankl. São Paulo: Paulus.

Aquino, T. A. A., Correia, A. P. M., Marques, A. L. C., Souza, C. G., Freitas, H. C. A., Araújo, I. F., Dias, P. S. A., \& Fernandes, W. (2009). Atitude religiosa e sentido de vida: Um estudo correlacional. Psicologia: Ciência e Profissão, $29,228-243$

Aquino, T. A. A., Gouveia, V. V., Pontes, A. M., Pereira, G. A., \& Fernandes, A. S. (no prelo). Questionário de Sentido de Vida: Evidências de sua validade fatorial e consistência interna. Psicologia: Ciência e Profissão.

Aquino, T. A. A., Gouveia, V. V., Silva, S. S., \& Aguiar, A. A. (2013). Escala de Atitudes Religiosas, versão expandida (EAR-20): Evidências de validade. Avaliação Psicológica, 12,109-119.

Amâncio, D. C. O., Nunes, M. T. C. C., \& Cardoso, M. A. G. (2010). Resposta imune humoral frente à situação de estresse. XIV Encontro Latino Americano de Iniciação Científica e X Encontro Latino Americano de Pós-Graduação. 
Amatuzzi, M. M. (2000). Desenvolvimento religioso: Uma hipótese psicológica. Estudos de Psicologia (PUC-Campinas), 17, 15-30. http://dx.doi.org/10.1590/S0103-166X2000000100002

Balestieri, F. M. P. (2005). Imunologia. São Paulo: Malone.

Bower, G. H. (1981). Mood and memory. American Psychologist, 36, 129-148. http://dx.doi.org/10.1037/0003066X.36.2.129

Brasil. Ministério da Saúde. (2012). Secretária de Vigilância em Saúde. Departamento de DST, AIDS e Hepatites Virais. Boletim Epidemiológico - AIDS e DST.

Byrne, B. M. (2010). Structural equation modeling with Amos: Basic concepts, applications, and programming (2를. New York: Taylor and Francis.

Cotton, S., Puchalski, C. M., Sherman, S. N., Mrus, J. M., Peterman, A. H., Feinberg, J., Pargament, K. I., Justice, A. C., Leonard, A. C., \& Tsevat, J. (2006). Spirituality and religion in patients with HIV/AIDS. Journal of General Internal Medicine, 21, 5-13. http://dx.doi.org/10.1111/j.1525-1497.2006.00642.x

Diener, E. \& Emmons, R. (1984). A The independence of positive and negative affect. Journal of Personality and Social Psychology, 47, 1105-1117. http://dx.doi.org/10.1037/0022-3514.47.5.1105

Diniz, A. C. \& Aquino, T. A. A. (2009). A relação da religiosidade com as visões de morte. Religare, 1, 90-105.

Espírito Santo, C. C. (2011). Expressões da espiritualidade em pessoas que vivem com HIV/AIDS a partir das representações sociais acerca da síndrome: Implicações para o cuidado de enfermagem. Dissertação de Mestrado. Departamento de Enfermagem, Universidade do Estado do Rio de Janeiro, RJ.

Ferreira, D. C., Favoreto, C. A. O., \& Guimarães, M. B. L. (2012). A influência da religiosidade no conviver com o HIV. Interface, 16, 383-394. http://dx.doi.org/10.1590/S1414-32832012005000019

Frankl, V. E. (1992). Teoría y terapia de las neurosis: Iniciación a la logoterapia y análisis existencial. Madrid: Herder.

Frankl, V. E. (2008). Em busca de sentido: um psicólogo no campo de concentração (Trad. Walter O. Schlupp \& Carlos C. Aveline) (25a ed.). São Leopoldo, RS: Sinodal / Petrópolis, RJ: Vozes.

Frankl, V. E. (2009). A presença ignorada de Deus (Trad. W. O. Schlupp \& H. H. Reinhold) (11ª ed.). São Leopoldo, RS: Sinodal/Petrópolis, RJ: Vozes.

Frankl, V. E. (2010). Psicoterapia e sentido da vida: Fundamentos da logoterapia e análise existencial (Trad. Alípio Maia de Castro) (5ª ed.). São Paulo: Quadrante.

Frankl, V. E. (2011). A vontade de sentido: Fundamentos e aplicações da logoterapia (Trad. Ivo Studart Pereira). São Paulo: Paulus.

Frankl, V. E. (2012). Logoterapia e análise existencial: textos de seis décadas. Rio de Janeiro: Forense Universitária.

Fryback, P. B. \& Reinert, B. R. (1999). Spirituality and people with potentially fatal diagnoses. Nursing Forum, 34, 13-22. http://dx.doi.org/10.1111/j.1744-6198.1999.tb00231.x

Geocze, L., Mucci, S., Marco, M. A., Nogueira-Martins, L. A., \& Citero, V. A. (2010). Qualidade de vida e adesão ao tratamento anti-retroviral de pacientes portadores de HIV. Revista de Saúde Pública, 44, 743-749. http://dx.doi. org/10.1590/S0034-89102010000400019

Hamilton, J. M., Kives, K. D., Micevski, V., \& Grace, S. L. (2003). Time perspective and health-promoting behaviour in a cardiac rehabilitation population. Behavioural Medicine, 28, 132-139. http://dx.doi.org/10.1080/08964280309596051

Kasser, T. \& Ahuvia, A. C. (2002). Materialistic values and well-being in business students. European Journal of Social Psychology, 32, 137-146. http://dx.doi.org/10.1002/ejsp.85

Kasser, T. \& Ryan, R. M. (1993). A dark side of the American dream: Correlates of financial success as a central life aspiration. Journal of Personality and Social Psychology, 65, 410-422. http://dx.doi.org/10.1037/0022-3514.65.2.410

Knapp, P. (Org.). (2004). Terapia cognitivo-comportamental na prática psiquiátrica. Porto Alegre: Artes Médicas.

Koenig, H. G., McCullough, M. E., \& Larson, D. B. (2001). Handbook of religion and health: A century of research reviewed. New York: Oxford University Press. http://dx.doi.org/10.1093/acprof:oso/9780195118667.001.0001

Leserman, J. (2008). Role of depression, stress, and trauma in HIV disease progression. Psychosomatic Medicine, 70 , 539-545. http://dx.doi.org/10.1097/PSY.0b013e3181777a5f

Linares, V. R., Burón, R. R., Rodríguez, J. A. P., López, L. J. G., \& Guadalupe, L. A. O. (2008). Psiconeuroinmunología: Conexiones entre sistema nervioso y sistema inmune. Suma Psicológica, 15, 115-142.

Litwinczuk, K. M. \& Groh, C. J. (2007). The relationship between spirituality, purpose in life, and well-being in HIV-positive persons. Journal of the Association of Nurses in AIDS care, 18, 13-22. http://dx.doi.org/10.1016/ j.jana.2007.03.004

Lotufo Neto, F. (2003). Religião, psicoterapia e saúde mental. In C. N. Abreu (Org.), Psicoterapias cognitiva e construtivista: Novas fronteiras da prática clínica (pp. 289-315). Porto Alegre: Artmed.

Lukas, E. (1989). Logoterapia: A força desafiadora do espírito (Trad. J. de Sá Porto). São Paulo: Edições Loyola/Santos, SP: Leopodianum Editora.

Lukas, E. (1990). Mentalização e saúde: A arte de viver e logoterapia (Trad. Helga Hinkenickel Reinhold). Petrópolis, RJ: Vozes.

Marques, M. C. C. (2003). A história de uma epidemia moderna - A emergência política da AIDS/HIV no Brasil. São Carlos, SP: Rima.

Mello, V. A., Segurado, A. A. C., \& Malbegier, A. (2010). Depression in women living with HIV: Clinical and psychosocial correlates. Archives of Women's Mental Health, 13,193-199. http://dx.doi.org/10.1007/s00737-009-0094-1 
Moreira-Almeida, A., Lotufo Neto, F., \& Koenig, H. G. (2006). Religiousness and mental health: a review. Revista Brasileira de Psiquiatria, 28, 242-250. http://dx.doi.org/10.1590/S1516-44462006005000006

Nauert, R. (2011). Attitude about past influences health. PsychCentral. Acessado em 20 de março de 2013, disponível em http://psychcentral.com/news/2011/09/07/attitude-about-past-influences-health/29216.html

Oyanadel, C. \& Buela-Casal, G. (2011). La percepción del tiempo: Influencias en la salud física y mental. Universitas Psychologica, 10, 149-161.

Panzini, R. G. \& Bandeira, D. R. (2007). Coping (enfrentamento) religioso/espiritual. Revista de Psiquiatria Clínica, 34 , 126-135. http://dx.doi.org/10.1590/S0101-60832007000700016

Panzini, R. G. \& Bandeira, D. R. (2005). Escala de coping religioso-espiritual (escala CRE1): Elaboração e validação de construto. Psicologia em Estudo (Maringá), 10, 507-516.

Pergher, G. K., Grassi-Oliveira, R., Ávila, L. M., \& Stein, L. M. (2006). Memory, mood and emotion. Revista de Psiquiatria do Rio Grande do Sul, 28, 61-68. http://dx.doi.org/10.1590/S0101-81082006000100008

Remor, E. A. (2002). Aspectos psicossociais na era dos novos tratamentos da AIDS. Psicologia: Teoria e Pesquisa, 18, 283-287. http://dx.doi.org/10.1590/S0102-37722002000300007

Resende, M. C., Silva, R. M., Marques, T. P., \& Abreu, M. V. (2008). Coping e satisfação com a vida em adultos com AIDS. Psico-PUCRS, 39, 232-239.

Saldanha, A. A. W. (2003). Vulnerabilidade e construções de enfrentamento da soropositividade ao HIV por mulheres infectadas em relacionamento estável. Tese de doutorado não-publicada. Pós-Graduação em Psicologia, Faculdade de Filosofia, Ciências e Letras de Ribeirão Preto, Universidade de São Paulo, Ribeirão Preto, SP.

Saroglou, V. (2003). Spiritualité moderne: un regard de psychologie de la religion. Revue Théologique de Louvain, 34 , 473-504. http://dx.doi.org/10.2143/RTL.34.4.2017462

Seidl, E. M. F., Zannon, C. M. L. C., \& Tróccoli, B. T. (2005). Pessoas vivendo com HIV/AIDS: enfrentamento, suporte social e qualidade de vida. Psicologia: Reflexão e Crítica, 18, 188-195. http://dx.doi.org/10.1590/S010279722005000200006

Serrano-Villar, S., Sainz, T., Lee, S. A., Hunt, P. W., Sinclair, E., Shacklett, B, L., Ferre, A. L., Hayes, T. L., Somsouk, M., Hsue, P. Y., Van, N. M., Meinert, C. L., Lederman, M. M., Hatano, H., Jain, V., Huang, Y., Hecht, F. M., Martin, J. N., McCune, J. M., Moreno, S., \& Deeks, S. G. (2014). HIV-infected individuals with low CD4/CD8 ratio despite effective antiretroviral therapy exhibit altered T cell subsets, heightened CD8+ T cell activation, and increased risk of non-AIDS morbidity and mortality. PLoS Pathog, 10(5): e1004078. http://dx.doi.org/10.1371/journal.ppat.1004078

Silva, A. L. C. N., Waidman, M. A. P., \& Marcon, S. S. (2009). Adesão e não-adesão à terapia antirretroviral: As duas faces de uma mesma vivência. Revista Brasileira de Enfermagem, 62, 213-220. http://dx.doi.org/10.1371/journal. ppat. 1004078

Silva Filho, N., Chaves Júnior, J. C. S., \& Campos, D. (2012). Relações entre o funcionamento dos sistemas imunológico e psicológico em pacientes com HIV/SIDA. In J. L. P. Ribeiro, I. Leal, A. Pereira, \& S. Monteiro (Orgs.), Psicologia da Saúde: desafios à promoção da saúde em doenças crônicas (pp. 113-119). Lisboa: Placebo.

Steger, F. M., Frazier, P., Oishi, S., \& Kaler, M. (2006). The Meaning in Life Questionnaire: Assessing the presence of and search for meaning in life. Journal of Counseling Psychology, 53, 80-93. http://dx.doi.org/10.1037/0022-0167.53.1.80

Teixeira, F. (2005). O potencial libertador da espiritualidade e da experiência religiosa. In M. M. Amatuzzi (Org.). Psicologia e espiritualidade (pp. 13-30). São Paulo: Paulus.

Terrin, A. N. (1998). A doença? Síndrome de desarmonia do espírito tratado sobre religiões antigas e novas. In F. Dal Pino, R. Fabris, E. Fizzotti, U. Galimberti, B. Häring, A. Langella, J. Ries, B. Secondin, G. Sovernigo, A. N. Terrin, \& S. Ubbiali, S. (Orgs.), Liturgia e terapia: A sacramentalidade a serviço do homem na sua totalidade (pp. 181-234). São Paulo: Paulinas.

Unaids, Joint United Nations Programme on HIV/AIDS. (2012). Global report: UNAIDS report on the global AIDS epidemic 2012. Geneva. World Healt Organization.

Veronesi, R. \& Focaccia, R. (2010). Tratado de Infectologia (4⿳⺈-e ed.) (Vol. 2). São Paulo: Atheneu.

Woods, T. E., Antoni, M. H., Ironson, G. H., \& Kling, D. W. (1999). Religiosity is associated with affective and immune status in symptomatic HIV-infected gay men. Journal of Psychosomatic Research, 46, 165-176. http://dx.doi. org/10.1016/S0022-3999(98)00078-6

\footnotetext{
Autores: Universidade Federal da Paraíba (UFPB). Federal da Paraíba (UFPB). pela Universidade Federal Fluminense (UFF).

Endereço para correspondência:

Alisson de Meneses Pontes

Rua Alto Casteliano, 530.- Bairro Santo Antônio

58701-070 Patos, PB, Brasil

Recebido em: 18.05 .14

Aceito em: 10.10.14
}

Alisson de Meneses Pontes - Professor do curso de Psicologia das Faculdades Integradas de Patos (FIP). Mestre em Ciências das Religiões pela Universidade Federal da Paraíba e doutorando em Psicologia Clínica na Universidade Católica de Pernambuco (UNICAP).

Thiago Antonio Avellar De AQuino - Professor Adjunto da Universidade Federal da Paraíba (UFPB). Doutor em Psicologia Social pela

VALDINEY Veloso GouVEIA - Doutor na Universidade Federal da Paraíba.

PATRÍCIA NUNES DA FONSÊCA - Professora Adjunta da Universidade Federal da Paraíba (UFPB). Doutora em Psicologia Social pela Universidade

Berta LÚCIA PINHEIRo KLÜPPEL - Professora Associada da Universidade Federal da Paraíba (UFPB). Doutorado em Patologia Experimental 